\title{
Use of macrolides in mother and child and risk of infantile hypertrophic pyloric stenosis: nationwide cohort study
}

\author{
(c) (1) (8)
}

\author{
Marie Lund PhD student ${ }^{1}$, Björn Pasternak postdoctoral fellow ${ }^{1}$, Rie B Davidsen statistician ${ }^{1}$, Bjarke \\ Feenstra senior researcher ${ }^{1}$, Camilla Krogh paediatric resident ${ }^{1}$, Lars J Diaz statistician ${ }^{1}$, Jan \\ Wohlfahrt chief statistician $^{1}$, Mads Melbye professor ${ }^{12}$
}

'Department of Epidemiology Research, National Health Surveillance and Research, Statens Serum Institut, Artillerivej 5, 2300 Copenhagen S, Denmark; ${ }^{2}$ Department of Medicine, Stanford School of Medicine, Stanford, CA, USA

\begin{abstract}
Objective To assess the association between use of macrolide antibiotics in mothers and infants from pregnancy onset until 120 days after birth and infantile hypertrophic pyloric stenosis (IHPS).

Design Nationwide register based cohort study.

Setting Denmark, 1996-2011.

Participants 999378 liveborn singletons and linked individual level information on macrolide prescriptions (maternal use during pregnancy, $\mathrm{n}=30$ 091; maternal use after birth, $\mathrm{n}=21557$; use in infants, $\mathrm{n}=6591$ ), surgery for IHPS, and potential confounders.

Main outcome measures Surgery for IHPS by three categories of macrolide use: in mothers during pregnancy, in mothers after birth, and in infants after birth.

Results 880 infants developed IHPS (0.9 cases per 1000 births). Compared with infants with no use of macrolides, the adjusted rate ratio for IHPS in infants with use of macrolides during days 0 to 13 after birth was 29.8 (95\% confidence interval 16.4 to 54.1 ) and during days 14 to 120 was 3.24 ( 1.20 to 8.74 ); the corresponding absolute risk differences were 24.4 (95\% confidence interval 13.0 to 44.1 ) and 0.65 (0.06 to 2.21 ) cases per 1000 infants exposed to macrolides, respectively. The rate ratio for maternal use of macrolides for days 0 to 13 after birth was 3.49 (1.92 to 6.34 ) and for days 14 to 120 was 0.70 (0.26 to 1.90$)$; the corresponding absolute risk differences were 2.15 (0.82 to 4.64$)$ and $-0.11(-0.26$ to 0.31$)$. The rate ratios for maternal use of macrolides during pregnancy were 1.02 (0.65 to 1.59$)$ for weeks 0 to 27 and 1.77 ( 0.95 to 3.31 ) for weeks 28 to birth; the corresponding absolute risk differences were $0.01(-0.31$ to 0.50$)$ and 0.67 ( -0.06 to 2.02$)$.
\end{abstract}

Conclusions Treatment of young infants with macrolide antibiotics was strongly associated with IHPS and should therefore only be administered if potential treatment benefits outweigh the risk. Maternal use of macrolides during the first two weeks after birth was also associated with an increased risk of IHPS. A possible association was also found with use during late pregnancy.

\section{Introduction}

Macrolide antibiotics are widely used in the treatment of upper and lower respiratory tract infections and sexually transmitted diseases. Infantile hypertrophic pyloric stenosis (IHPS) is the most common condition requiring surgery during the first months of life, where infants, typically between 3 and 12 weeks old, present with progressively worsening vomiting due to hypertrophy of the pyloric muscle. Following a decline in incidence of this condition in many countries from the beginning of the 1990s, the current estimate for IHPS is around 1 to 2 per 1000 births. $^{1-5}$

IHPS has been associated with genetics,${ }^{6-8}$ boys,,${ }^{9}{ }^{10}$ family history, ${ }^{24}$ and birth order (first born), ${ }^{11}$ and with environmental factors such as bottle feeding. ${ }^{12}{ }^{13}$ Moreover, infants treated with the macrolide erythromycin during the first two weeks after birth are at increased risk of IHPS. ${ }^{14-16}$ It is, however, unclear whether the increased risk associated with macrolide use extends beyond these two weeks or whether the infantile gut is particularly susceptible during the two weeks after birth. As an up to 10-fold increase in risk of IHPS has been reported after use of macrolides in infants it has been debated whether the risk might also be increased by maternal use. Although the evidence is sparse, macrolides are excreted in breast milk at low levels 
and are thus generally considered safe during breast feeding. ${ }^{17-20}$ A study of maternal postnatal macrolide use reported twofold to threefold increased odds of IHPS, but the association was not statistically significant and was only based on three exposed cases. ${ }^{21}$ Studies of macrolide use during pregnancy and IHPS report heterogeneous results but suggest a possible modestly increased risk with use in late pregnancy. ${ }^{1622-24}$

In a nationwide register based cohort study of approximately one million liveborn singletons and their mothers, we assessed the risk of IHPS after exposure to macrolides in utero and after postnatal use in both mothers and infants.

\section{Methods}

We identified a cohort of all liveborn singletons in Denmark (1 January 1996 to 31 December 2011) using the Danish Civil Registration System. ${ }^{25}$ The Civil Registration System was established in April 1968 and contains individual level information on demographics, vital status, and parent-child links for all Danish citizens. We obtained information on the outcome of IHPS from the national patient register, ${ }^{26}$ by use of diagnostic codes and surgery codes. Diagnostic codes have been coded using ICD-10 (international classification of diseases, 10th revision) since 1994 and surgery codes using the Nordic classification of surgical procedures since 1996. We obtained information on antibiotic use from the prescription drug register. ${ }^{27}$ This register contains information on all filled prescriptions at Danish pharmacies since 1995, including date of filling the prescription and anatomical therapeutic chemical (ATC) code. We obtained information on prescriptions filled by cohort mothers or for their children from pregnancy onset until 120 days after birth for any macrolide (ATC code J01FA), and also for penicillin V (ATC code J01CE02) and amoxicillin (ATC code J01CA04) for sensitivity analyses. From the medical birth register ${ }^{28}$ we obtained information on covariates related to pregnancy and obstetrical outcome. Finally, for additional analyses of robustness, from the national patient register we obtained information on infant admissions to hospital for all causes and maternal admissions for infectious diseases. A unique personal identification number enabled linkage of individual level information between the different nationwide registers. (The supplementary file contains a basic description of the Danish nationwide registers and diagnostic codes used to define admissions to hospital for infectious diseases.)

\section{Use of macrolides}

Macrolide use was defined as filling a prescription for any macrolide (antibiotics are not available over the counter in Denmark). We assessed maternal use of macrolides during pregnancy, maternal use after birth, and use in infants after birth during predefined exposure windows (chosen a priori on the basis of the literature ${ }^{14-1621-24}$ ): gestational weeks 0-27 and weeks 28 to birth, and days 0-13 and 14-120 after birth. In case of multiple prescriptions during pregnancy, we used the latest prescription to define the exposure window. For postnatal macrolide use, once participants had used macrolides they remained in the exposed group throughout follow-up, and in case of multiple prescriptions, we used the first prescription to define the exposure window.

\section{Surgery for IHPS}

We defined IHPS cases as infants with a surgical code of pyloromyotomy during the first 120 days after birth (Nordic classification of surgical procedures KJDH60 or KJDH61); IHPS is rare at older ages. ${ }^{10}$ The IHPS index date was defined as date of admission to hospital for IHPS surgery or, if a distinct admission or outpatient visit including a diagnostic code for IHPS (ICD-10: Q40.0, DK31.1, DK31.1A, DK31.3, DK31.8B) preceded the date of admission to hospital for IHPS surgery, we used the date of the record containing the diagnostic code as the index date.

\section{Statistical analyses}

We studied the association between macrolide use and risk of IHPS by (incidence) rate ratios in a log linear Poisson regression model comparing exposed and unexposed infants followed from birth until 120 days after birth, death, emigration, disappearance, IHPS, or 31 December 2011, whichever occurred first. Postnatal exposures were time dependent, and cohort members contributed with person years of follow-up in the unexposed group until first macrolide prescription, after which they contributed with person years of follow-up in the exposed group for the remaining part of the follow-up time. We carried out analyses using SAS software (version 9.3), and all tests were performed as log likelihood ratio tests.

For each exposure window we defined confounders a priori on the basis of the literature and biological considerations. For all main analyses these were birth order, sex, calendar period, and current age of the infant. We additionally included gestational age at birth, being small for gestational age, caesarean section, major congenital malformations, and maternal smoking during pregnancy as adjustment variables in the main analyses of exposure from maternal and infant use after birth, whereas for maternal use during pregnancy we considered these as potential mediators and excluded them from the main model. If a covariate could be associated with macrolide use and be a cause of the outcome, we considered it to be a potential confounder; however, if the covariate could be found on the causal pathway between macrolide use and outcome, we considered it a potential mediator. We evaluated potential mediators and potential confounders (for all categories of macrolide use: seasonality and maternal age) by a change of estimate approach (10\% cut off) with separate inclusion in the main analyses. We additionally adjusted rate ratios for any previous macrolide use: maternal use during gestational weeks 0 to 27 was not further adjusted, maternal use during gestational weeks 28 to birth was adjusted for any use during gestational weeks 0 to 27 , maternal use after birth was adjusted for use in pregnancy and for use in infants preceding the maternal use, and use in infants after birth was adjusted for use in pregnancy and maternal use preceding the use in infants. For analyses of filled prescription for penicillin or amoxicillin as exposure, adjustment for potential confounders was performed similarly to the main analyses except for adjustment for preceding antibiotic use where estimates were adjusted for preceding use of penicillin $\mathrm{V}$, amoxicillin, or macrolides. Unless otherwise stated, use of the term rate ratio in the paper implies adjusted rate ratio-adjusted. Mother-infant pairs could contribute to multiple exposure windows, and infants could be exposed in six different exposure windows: four through maternal use of macrolides and two through use in the infants themselves. To tackle the potential mediating effect of postnatal macrolide use on the association between maternal use during pregnancy and IHPS, we performed additional sensitivity analyses with censoring at any postnatal use. Similarly, we assessed the association between maternal postnatal use and IHPS with censoring at any macrolide use in the infant. We included interaction terms to evaluate effect modification by covariates. In each specific analysis we only included infants with full information on covariates. (See supplementary table 1 for full details of the covariates.) 
We calculated the absolute risk difference of IHPS among cohort members who did or did not use macrolides as the difference between the cumulative incidences in the two groups at 120 days. The cumulative incidence among cohort members who did not use macrolides was estimated based on the observed overall age specific rates, and the cumulative incidence among cohort members who used macrolides was estimated based on the observed overall age specific rates, the adjusted rate ratios, and the distribution of macrolide use in cohort members from 0 to 120 days after birth. The $95 \%$ confidence interval for an absolute risk difference was estimated based on 10000 simulations of the adjusted $\log$ (rate ratio) assuming that the $\log$ (rate ratio) was normal distributed, with mean and standard error taken as the estimator and standard error for the $\log$ (rate ratio) from the Poisson regression analysis behind the main results (see figure). For each of the 10000 simulations of the $\log$ (rate ratio) we calculated the absolute risk difference and estimated the associated $95 \%$ confidence intervals as the $2.5 \%$ and $97.5 \%$ centiles in the simulated distribution of absolute risk difference.

\section{Results}

The cohort included a total of 999378 liveborn singletons; the mothers of 30091 (3.0\%) had used macrolides during pregnancy and 21557 (2.2\%) had used macrolides from birth until 120 days after birth, and $6591(0.6 \%)$ of the infants had used macrolides from days 0 to 120 after birth (table $1 \Downarrow$ ). Table $2 \Downarrow$ shows the characteristics of the study cohort. During the study period, macrolide use decreased in the mothers during pregnancy, whereas maternal use and use in infants after birth was relatively stable over time. Infants exposed to macrolides, through maternal or own use, tended to have mothers who were younger and more often smokers. Infants prescribed macrolides were less often preterm.

\section{Rate ratios of IHPS by macrolide use}

During follow-up, 880 infants developed IHPS ( 0.9 cases per 1000 births). The figure $\Downarrow$ shows the rate ratios of IHPS according to macrolide use. The rate ratios for the association between macrolide use in infants and IHPS were 29.8 (95\% confidence interval 16.4 to 54.1) for days 0 to 13 after birth and 3.24 (1.20 to 8.74 ) for days 14 to 120 . The corresponding absolute risk differences per 1000 exposed infants were 24.4 (95\% confidence interval 13.0 to 44.1 ) for days 0 to 13 and 0.65 ( 0.06 to 2.21 ) for days 14 to 120 . The rate ratios for postnatal maternal use were 3.49 (1.92 to 6.34) for days 0 to 13 and 0.70 ( 0.26 to 1.90 ) for days 14 to 120 ; the corresponding absolute risk differences per 1000 exposed infants were 2.15 (0.82 to 4.64) for days 0 to 13 and -0.11 ( -0.26 to 0.31 ) for days 14 to 120. The rate ratios for maternal use of macrolides during pregnancy were 1.02 ( 0.65 to 1.59 ) for weeks 0 to 27 and 1.77 ( 0.95 to 3.31 ) for weeks 28 to birth; the corresponding absolute risk differences per 1000 exposed infants were $0.01(-0.31$ to 0.50 ) for weeks 0 to 27 and 0.67 ( -0.06 to 2.02 ) for weeks 28 to birth.

\section{Preplanned sensitivity analyses}

Among infants who were prescribed macrolides and subsequently developed IHPS, the median time from date of a filled prescription to IHPS was 24 days (range 9-59 days; see supplementary figure 1). We estimated rate ratios of IHPS according to time since a filled prescription for macrolides to explore a differential effect ( $\leq 2$ weeks $v>2$ weeks) for the three categories of macrolide use where a significant association was observed and the rate ratios did not differ significantly (see supplementary table 3 ).

We found no indication of effect modification according to infants' sex and congenital malformations (although based on small numbers in each subgroup), except for the analysis of maternal use of macrolides during days 0 to 13 after birth, where the rate ratio was significantly higher in infants with congenital malformations than in infants without such malformations (see supplementary table 3 ).

We assessed several additional variables as potential confounders or mediators. The inclusion of maternal age and seasonality in all models and perinatal outcomes, congenital malformations, and smoking in models of maternal use of macrolides during pregnancy did not notably change the estimates (see supplementary table 4).

To evaluate possible confounding by indication we examined the association between use of penicillin or amoxicillin and IHPS. We observed no significant associations (table $3 \Downarrow$ ).

Information about drugs administered during hospital stay was not available. To remove the potential influence of possible use of antibiotics during hospital stay, we performed analyses with censoring of infants who were admitted to hospital for any cause for five days or more from postpartum day 5 or later, and mothers admitted to hospital due to infection during pregnancy or post partum. Despite a considerable reduction in the number of cases in these analyses, the estimates were largely similar to the main analyses (see supplementary table 5).

\section{Additional sensitivity analyses}

To assess whether the borderline significant association between maternal use of macrolides during gestational weeks 28 to birth and IHPS was driven by postnatal use of macrolides, we performed analyses with censoring at any postnatal use of macrolides, yielding a rate ratio of 1.54 (95\% confidence interval 0.77 to 3.09). To assess whether the significant association observed for maternal use of macrolides during days 0 to 13 was driven by use in infants, we performed analyses with censoring at use of macrolides in infants, yielding a rate ratio of 2.38 (1.13 to 5.01).

To explore the window of maximal susceptibility to postnatal use we subdivided the period from days 14 to 120 . The rate ratios for use in infants were 2.51 (0.62 to 10.2) for days 14 to 27 and 4.53 (1.12 to 18.2) for days 28 to 120 . For maternal use, the rate ratios were 1.10 ( 0.40 to 2.97$)$ for days 14 to 27 and non-estimable (no exposed cases) for days 28 to 120 .

To explore the possibility of an effect of specific macrolide subtypes, we assessed rate ratios of IHPS for the six categories of use according to macrolide subtype (see supplementary table 6). Although these results should be interpreted with caution owing to the small number of events (or no events) in some of the exposure windows and owing to these analyses being performed as additional sensitivity analyses, estimates seemed to be largely similar across the various macrolide subtypes. For maternal use during gestational weeks 28 to birth, all cases of IHPS were exposed to erythromycin, yielding a rate ratio of 2.24 (1.20 to 4.18), and for maternal use during gestational weeks 0 to 27, six cases were exposed to roxithromycin, yielding a rate ratio of 2.51 (1.12 to 5.60). To assess whether these associations were driven by postnatal use of macrolides, we performed additional analyses with censoring at any postnatal use of macrolides (infant or maternal), which attenuated the rate ratios to 1.98 ( 0.82 to 4.77 ) and 1.91 (0.95 to 3.83$)$, respectively. For use of macrolides in infants, all exposed IHPS cases were prescribed erythromycin. 
To evaluate whether the main results were influenced by a mother contributing more than one child to the cohort, we performed analyses where only the first child from the mother was included in the analyses (remaining cases of IHPS in analyses: 617 for maternal use during pregnancy, 601 for maternal use after birth, and 495 for use in infants after birth). This resulted in similar results (data not shown).

We performed sensitivity analyses to evaluate the impact of the breastfeeding pattern of Danish women on the association between maternal use of macrolides after birth and IHPS. For instance, assuming that $90 \%$ of infants are breast fed, the rate ratio would increase by $40 \%$ (see analyses in the supplementary appendix).

\section{Discussion}

In this nationwide cohort, macrolide use in infants was associated with a strongly increased risk of infantile hypertrophic pyloric stenosis (IHPS), including a 30 -fold increased risk with use during the first two weeks after birth and a lower, but significantly increased threefold risk with use on days 14 to 120. Similarly, the risk of IHPS was increased more than threefold with maternal use of macrolides during the first two weeks after birth, but not increased with macrolide use thereafter. We found no evidence of an association between IHPS and maternal use of macrolides during gestational weeks 0 to 27 , but a possible modest association with use during weeks 28 to birth.

\section{Meaning of results and comparison with other studies}

Previous studies on the association between use of macrolides in infants and IHPS report up to a 10 -fold increased risk of the condition with use of erythromycin in infants within the first two weeks after birth, ${ }^{14}{ }^{16}$ whereas we found a 30 -fold increased risk. However, comparing absolute risk differences, which take into account the baseline risk of IHPS in the studied population, we found an excess of 24.4 cases per 1000 exposed cohort members compared with 15 to 30 excess cases per 1000 exposed cohort members in the two previous studies (based on the reported relative risks and overall incidence rates $\left.{ }^{14}{ }^{16}\right)$. In contrast with the previous studies we also found an association with use of macrolides in infants during days 14 to 120 after birth and IHPS, albeit a substantially weaker association. Moreover, investigating the strong association between IHPS and use of macrolides in infants, we found it to be better described by time of filled prescription rather than by time since filled prescription. Viewed together with the analysis of maternal postnatal use showing an increased risk for days 0 to 13 only, these data suggest a specific time window where the infant gut is particularly susceptible. This notion is further supported by our exploratory analysis showing that the risk of IHPS was substantially lower with use in infants during days 14 to 27 after birth than during days 0 to 13 .

We found a significant, more than threefold increased risk of IHPS associated with maternal macrolide use during the first two weeks after birth. The observed association was attenuated but remained significant when concomitant macrolide use in infants was taken into account. In line with our results, a previous smaller study reported a twofold to threefold increased risk of IHPS associated with maternal macrolide use after birth, although the results did not reach statistical significance. ${ }^{21}$ For maternal use during days 14 to 120 we did not find evidence of an association with IHPS. The lack of information on breastfeeding status could, however, have led to two different biases influencing this estimate. Firstly, it is possible that women who were prescribed macrolides were less likely to have breast fed owing to the underlying infection or fear of drug transfer to the infant. With the measurable levels of macrolides in breast milk being much smaller than the standard regimens used in infants, ${ }^{20}$ macrolide use has generally been considered safe in lactating women. Together with the focus on the beneficial effects of breast feeding, ${ }^{29}$ this argues against the likelihood of major bias due to cessation of breast feeding for the group of women in question: still, it should be taken into account in the interpretation of the results. The second possible bias relates to the proportion of women breast feeding in Denmark. More than 95\% of Danish mothers have been reported to take up breast feeding immediately after birth, ${ }^{29}$ and in 2000 approximately $75 \%$ of infants were breast fed at 4 months of age. ${ }^{30}$ Because we assumed that children of mothers who were prescribed macrolides after birth were exposed to macrolides through breast milk, some of the infants defined as exposed through maternal breast milk in our study were probably not exposed, leading to conservative estimates. Consequently, the magnitude of the association is possibly greater for exposure on days 0 to 13 , whereas an increased risk with exposure on days 14 to 120 might have been masked. We thus performed sensitivity analyses to evaluate the impact of the pattern of breast feeding in Danish women on the association between maternal macrolide use after birth and IHPS. For instance, assuming that $90 \%$ of infants were breast fed, the rate ratio would increase by $40 \%$.

With respect to gestational macrolide use, our study adds to the data suggesting no association between IHPS and macrolide use in the first two trimesters of pregnancy but a possible modest association with use in late pregnancy. No study has ruled out with certainty an association for use in late pregnancy, but three studies have reported point estimates between 0.60 and 2.45 (with the upper limits of the confidence intervals between 1.64 and 11.1). ${ }^{1622} 24$ Our study expands on this evidence by showing that the observed, albeit statistically non-significant, association with use in late pregnancy was not driven by postnatal macrolide use. While these results may point towards caution in using macrolides in late pregnancy, the totality of the findings needs further exploration-for example, using meta-analytical methods. However, any increase in risk would remain small in absolute terms.

To eliminate any influence from congenital malformations, we performed a sensitivity analysis according to the presence of malformations for the three categories of macrolide use where a significant association was observed. This showed that the majority of IHPS cases exposed to macrolides did not have concomitant malformations and that the associations observed in the primary analyses were consistent in infants without malformations. Moreover, in additional sensitivity analyses we explored the risk of IHPS according to specific macrolide subtype (for all six exposure windows). Although numbers were small, results were largely similar across the various subtypes. Although the power of the present study does not allow for inferences about any differences in risk profile according to specific macrolide subtype, no major differences were apparent across the individual drugs for the results according to specific macrolide subtype. We found a statistically significant more than twofold increased risk of IHPS associated with use of roxithromycin during gestational weeks 0 to 27 and with erythromycin during gestational weeks 28 to birth. However, when postnatal macrolide use was taken into account the associations were attenuated and no longer significant.

Erythromycin and azithromycin have been shown to be effective in the treatment of small bowel dysmotility and gastroparesis, ${ }^{31-33}$ 
with the effect in part explained by an agonistic action on motilin receptors. ${ }^{34-36}$ With a high concentration of motilin receptors in the gastric antrum ${ }^{37}$ - that is, the part of the stomach where the pyloric sphincter is also found - a possible biological mechanism behind the observed association could be a prokinetic effect of macrolides on the gastrointestinal smooth muscle, causing spasm of the pyloric muscle and subsequent IHPS through work induced hypertrophy.

\section{Strengths of this study}

The strengths of this study include the use of nationwide registers, which minimise recall and selection bias and allow independent assessment of exposure and outcome. In this large, nationwide cohort study, issues of generalisability with respect to, for instance, social class, should be of less concern than in studies based on data from health maintenance organisations. The Danish Civil Registration System is considered close to complete, including the mother-infant linkages. ${ }^{25}$ Ascertainment of macrolide use based on filled prescriptions maximised correctness of specific drug information. Any non-adherence to the drug would bias the association towards the null. Since users of multiple macrolide prescriptions were rarely observed among exposed cases with IHPS, it is unlikely that any major bias from cumulative use of macrolides exists. For the outcome of IHPS, surgery codes from the national patient register are generally considered accurate and well recorded. ${ }^{38}$ Various arguments exist against any major influence from bias due to the drug being prescribed because of symptoms attributable to the outcome studied: the observed significant associations did not vary according to time since macrolide prescription, and the median time from prescription of macrolide for infants to IHPS was 24 days (range 9-59); IHPS is a medical emergency in which infants progressively develop severe vomiting, and following diagnosis and fluid resuscitation, surgery is instituted promptly ${ }^{39}$ and the typical time course from symptom onset to surgery is usually less than a week ${ }^{40}$; and finally, to set the earliest possible index date for IHPS we used the date of admission with a diagnostic code for IHPS if this date preceded the date of admission for IHPS surgery.

\section{Limitations of this study}

The limitations of the study include the lack of information on the indication for the prescribed macrolide. In Denmark, where penicillin is first line treatment for most upper and lower respiratory tract infections, the main indications for macrolides are eradication therapy for Helicobacter pylori, treatment of sexually transmitted diseases, and respiratory tract infections in case of penicillin allergy or atypical pneumonia. In infants, macrolides are specifically used for pertussis infections. The drug prescription registry does not contain information on drugs prescribed during hospital stay. However, sensitivity analyses with censoring at maternal admission for infectious diseases during pregnancy or after birth and at infant admission for any cause after birth did not change the conclusions. Assuming a conservative approach, our finding of a rate ratio of 1.35 from maternal use of penicillin or amoxicillin during days 0 to 13 after birth indicates that some influence from infection or other factors associated with antibiotic use may have been present for this specific exposure category. For pregnancy use, our finding of no increased risk of IHPS associated with exposure from maternal use of penicillin or amoxicillin argues against any major confounding by indication. The indication, frequency, and range of prescriptions for penicillin or amoxicillin and macrolides are only in part overlapping, which should be kept in mind in the interpretation of the results of confounding by indication.

\section{Clinical implications and conclusions}

Whether an absolute risk increase of 24.4 cases of IHPS per 1000 infants exposed to macrolides within the first two weeks of life should prompt doctors to withhold the use of macrolides in infants of this age, depends on the indication for treatment. Pertussis infection in infants is associated with serious complications and is potentially fatal if untreated. In infants younger than 6 weeks, the macrolides azithromycin and clarithromycin are the only treatment alternatives for pertussis according to recommendations from the UK Health Protection Agency and the US Centers for Disease Control and Prevention; no other agents are recommended for this age group. ${ }^{41}{ }^{42}$ It seems probable that the absolute excess risk of IHPS, a condition treatable by a relatively uncomplicated surgical procedure, is outweighed by the risks of pertussis left untreated. Conversely, if effective and safe treatment alternatives exist (for example, for other infections) or if a clear and pertinent indication for treatment is missing, macrolides are best avoided in young infants. Although the absolute risks for IHPS were smaller for maternal use of macrolides when the infant was less than 14 days old and for use in infants during days 14 to 120 after birth, they should still be considered when treatment decisions are made. Most studies, including ours, implicate erythromycin; however, no study has been able to rule out associations with other macrolide agents with certainty. Therefore, a cautious prescription practice acknowledging the possibility of a class effect seems warranted pending additional data.

Contributors: ML, BP, RBD, BF, CK, JW, and MM conceived and designed the study. RBD, LJD, and JW did the statistical analysis. ML, $\mathrm{BP}, \mathrm{RBD}, \mathrm{BF}, \mathrm{CK}, \mathrm{JW}$, and MM analysed and interpreted the data. ML drafted the manuscript. BP, RBD, BF, CK, LJD, JW, and MM critically revised the manuscript for important intellectual content. $M L, B P$, and BF obtained funding. JW and MM supervised the study. MM is the guarantor.

Funding: ML was funded by a PhD study grant from the University of Copenhagen, BP by a grant from the Danish Medical Research Council (grant No 11-115854), and BF by an Oak Foundation fellowship (grant No OCAY-12-319). The funding agencies had no role in the design and conduct of the study; collection, management, analysis, and interpretation of the data; and preparation, review, or approval of the manuscript.

Competing interests: All authors have completed the ICMJE uniform disclosure form at http://www.icmje.org/coi_disclosure.pdf and ML declares support from the University of Copenhagen, BP from the Danish Medical Research Council, BF from the Oak Foundation, and the remaining coauthors declare no support from any organisation for the submitted work; all authors declare no financial relationships with any organisations that might have an interest in the submitted work in the previous three years; BF and MM have a patent WO 2013037371 A1 pending and the remaining authors declare no other relationships or activities that could appear to have influenced the submitted work.

Ethical approval: This study was approved by the Danish Data Protection Agency. Ethical approval is not required for register based research in Denmark.

Data sharing: No additional data available.

Transparency: All authors had full access to all of the data in the study and can take responsibility for the integrity of the data and the accuracy of the data analysis. The guarantor affirms that the manuscript is an honest, accurate, and transparent account of the study being reported; 


\section{What is already known on this topic}

Macrolide use in infants during the first two weeks of life has been associated with an increased risk of infantile hypertrophic pyloric stenosis (IHPS)

It is unclear if there is also an increased risk associated with use beyond two weeks or whether the infantile gut is particularly susceptible during the first two weeks after birth

It is not known whether maternal use of macrolides during late pregnancy and lactation increase the risk

\section{What this study adds}

Macrolide use in young infants was strongly associated with IHPS and should only be administered if potential treatment benefits outweigh the risk

Although the absolute risks for IHPS were smaller for maternal use of macrolides when infants were less than 2 weeks old and for use in infants during days 14 to 120 after birth, they should still be considered during decisions about treatment

A possible association exists with use during late pregnancy, but any real effect would be small in absolute terms

that no important aspects of the study have been omitted; and that any discrepancies from the study as planned have been explained.

1 De Laffolie J, Turial S, Heckmann M, Zimmer K-P, Schier F. Decline in infantile hypertrophic pyloric stenosis in Germany in 2000-2008. Pediatrics 2012;129:e901-6.

2 Krogh C, Fischer TK, Skotte L, Biggar RJ, Oyen N, Skytthe A, et al. Familial aggregation and heritability of pyloric stenosis. JAMA 2010;303:2393-9.

3 Nielsen JP, Haahr P, Haahr J. Infantile hypertrophic pyloric stenosis. Decreasing incidence. [In Danish.] Ugeskr Læger 2000;162:3453-5.

4 Persson S, Ekbom A, Granath F, Nordenskjöld A. Parallel incidences of sudden infant death syndrome and infantile hypertrophic pyloric stenosis: a common cause? Pediatrics 2001;108:e70.

5 Sommerfield T, Chalmers J, Youngson G, Heeley C, Fleming M, Thomson G. The changing epidemiology of infantile hypertrophic pyloric stenosis in Scotland. Arch Dis Child 2008:93:1007-11.

6 Everett KV, Chung EMK. Confirmation of two novel loci for infantile hypertrophic pyloric stenosis on chromosomes 3 and 5. J Hum Genet 2013;58:236-7.

7 Feenstra B, Geller F, Krogh C, Hollegaard MV, Gortz S, Boyd HA, et al. Common variants near MBNL1 and NKX2-5 are associated with infantile hypertrophic pyloric stenosis. Nat Genet 2012;44:334-7.

8 Feenstra B, Geller F, Carstensen L, Romitti PA, Körberg IB, Bedell B, et al. Plasma lipids, genetic variants near APOA1, and the risk of infantile hypertrophic pyloric stenosis. JAMA 2013;310:714-21

9 Adelstein P, Fedrick J. Pyloric stenosis in the Oxford Record Linkage Study area. J Med Genet 1976;13:439-48.

10 Krogh C, Gortz S, Wohlfahrt J, Biggar RJ, Melbye M, Fischer TK. Pre- and perinatal risk factors for pyloric stenosis and their influence on the male predominance. Am J Epidemiol 2012:176:24-31.

11 Still GF. Place-in-family as a factor in disease. Lancet 1927;795-800.

12 Krogh C, Biggar RJ, Fischer TK, Lindholm M, Wohlfahrt J, Melbye M. Bottle-feeding and the risk of pyloric stenosis. Pediatrics 2012;130:e943-9.

13 McAteer JP, Ledbetter DJ, Goldin AB. Role of bottle feeding in the etiology of hypertrophic pyloric stenosis. JAMA Pediatrics 2013:167:1143-9.

14 Cooper WO, Griffin MR, Arbogast P, Hickson GB, Gautam S, Ray WA. Very early exposure to erythromycin and infantile hypertrophic pyloric stenosis. Arch Pediatr Adolesc Med 2002;156:647-50

15 Honein MA, Paulozzi LJ, Himelright IM, Lee B, Cragan JD, Patterson L, et al. Infantile hypertrophic pyloric stenosis after pertussis prophylaxis with erythromcyin: a case review and cohort study. Lancet 1999:354:2101-5.

16 Mahon BE, Rosenman MB, Kleiman MB. Maternal and infant use of erythromycin and other macrolide antibiotics as risk factors for infantile hypertrophic pyloric stenosis. $J$ Pediatr 2001;139:380-4.

17 Kelsey JJ, Moser LR, Jennings JC, Munger MA. Presence of azithromycin breast milk concentrations: a case report. Am J Obstet Gynecol 1994;170:1375-6.

18 Lassman HB, Puri SK, Ho I, Sabo R, Mezzino MJ. Pharmacokinetics of roxithromycin (RU 965). J Clin Pharmacol 1988;28:141-52.

19 Sedlmayr T. Clarithromycin, a new macrolide antibiotic. Effectiveness in puerperal infections and pharmacokinetics in breast milk. [In German.] Geburtshilfe Frauenheilkd 1993:53:488-91.

20 Toxicology Data Network. Drugs and lactation database (LactMed). 2013. United States National Library of Medicine. http://toxnet.nlm.nih.gov/cgi-bin/sis/htmlgen?LACT).

21 Sorensen HT, Skriver MV, Pedersen L, Larsen H, Ebbesen F, Schonheyder HC. Risk of infantile hypertrophic pyloric stenosis after maternal postnatal use of macrolides. Scand $J$ Infect Dis 2003;35:104-6.

22 Cooper WO, Ray WA, Griffin MR. Prenatal prescription of macrolide antibiotics and infantile hypertrophic pyloric stenosis. Obstet Gynecol 2002;100:101-6.
23 Kallen BA, Otterblad OP, Danielsson BR. Is erythromycin therapy teratogenic in humans? Reprod Toxicol 2005;20:209-14.

24 Lin KJ, Mitchell AA, Yau WP, Louik C, Hernandez-Diaz S. Safety of macrolides during pregnancy. Am J Obstet Gynecol 2013;208:221-8.

25 Pedersen CB. The Danish Civil Registration System. Scand J Public Health 2011;39:22-5

26 Lynge E, Sandegaard JL, Rebolj M. The Danish National Patient Register. Scand J Public Health 2011:39:30-3.

27 Kildemoes HW. The Danish National Prescription Registry. Scand J Public Health 2011;39:38-41.

28 Knudsen LB, Olsen J. The Danish Medical Birth Registry. Dan Med Bull 1998;45:320-3.

29 Sundhedsstyrelsen [Danish Health and Medicines Authority]; Holk K, Kronborg H, Nilsson I. Håndbog i vellykket amning —en vejledning til sundhedspersonale. [In Danish.] Dansgaard B, ed; 2003. 2010. http://sundhedsstyrelsen.dk/publ/publ2003/vellykket amning.pdf.

30 Fogh A, Hallgreen KH, Salter A, Therkildsen I, Poulsen JW. Ammeundersøgelse 2000-2002, Amtsopgørelser. [In Danish.] Fagligt Forum for Amtssundhedsplejersker, 2003.

31 Chini P, Toskes PP, Waseem S, Hou W, McDonald R, Moshiree B. Effect of azithromycin on small bowel motility in patients with gastrointestinal dysmotility. Scand $J$ Gastroenterol 2012;47:422-7.

32 Di LC, Flores AF, Tomomasa T, Hyman PE. Effect of erythromycin on antroduodenal motility in children with chronic functional gastrointestinal symptoms. Dig Dis Sci 1994;39:1399-404

33 Janssens J, Peeters TL, Vantrappen G, Tack J, Urbain JL, De RM, et al. Improvement of gastric emptying in diabetic gastroparesis by erythromycin. Preliminary studies. NEngl J Med 1990;322:1028-31.

34 Broad J, Sanger GJ. The antibiotic azithromycin is a motilin receptor agonist in human stomach: comparison with erythromycin. Br J Pharmacol 2013;168:1859-67.

35 Peeters T, Matthijs G, Depoortere I, Cachet T, Hoogmartens J, Vantrappen G. Erythromycin is a motilin receptor agonist. Am J Physiol 1989;257:G470-4.

36 Sarna SK, Soergel KH, Koch TR, Stone JE, Wood CM, Ryan RP, et al. Gastrointestinal motor effects of erythromycin in humans. Gastroenterology 1991;101:1488-96.

37 Miller P, Roy A, St-Pierre S, Dagenais M, Lapointe R, Poitras P. Motilin receptors in the human antrum. Am J Physiol Gastrointest Liver Physiol 2000;278:G18-23.

38 Mosbech J, Jorgensen J, Madsen M, Rostgaard K, Thornberg K, Poulsen TD. The national patient registry. Evaluation of data quality. [In Danish.] Ugeskr Laeger 1995;157:3741-5.

39 Ranells JD, Carver JD, Kirby RS. Infantile hypertrophic pyloric stenosis: epidemiology, genetics, and clinical update. Adv Pediatr 2011;58:195-206.

40 Mackay AJ, MacKellar A. Infantile hypertrophic pyloric stenosis: a review of 222 cases. Aust N Z J Surg 1986;56:131-3.

41 Amirthalingam G; Pertussis Guidelines Group. HPA guidelines for the public health management of pertussis (updated October 2012). www.hpa.org.uk/webc/hpawebfile/ hpaweb_c/1287142671506.

42 Tiwari T, Murphy TV, Moran J; National Immunization Program, CDC. Recommended antimicrobial agents for the treatment and postexposure prophylaxis of pertussis: 2005 CDC Guidelines. MMWR 2005;54:1-16.

\section{Accepted: 24 February 2014}

\section{Cite this as: BMJ 2014;348:g1908}

This is an Open Access article distributed in accordance with the Creative Commons Attribution Non Commercial (CC BY-NC 3.0) license, which permits others to distribute, remix, adapt, build upon this work non-commercially, and license their derivative works on different terms, provided the original work is properly cited and the use is non-commercial. See: http://creativecommons.org/licenses/by-nc/3.0/. 


\section{Tables}

Table 1/ Use of any macrolide and subtypes in mothers and infants during pregnancy and up to 120 days after birth in a Danish cohort of 999378 singletons, 1996 to 2011

\begin{tabular}{lccc} 
& \multicolumn{3}{c}{ No (\% among ever users of any macrolide) $)^{*}$} \\
\cline { 2 - 4 } Drugs ever used & Maternal use during pregnancy & Maternal use $\mathbf{0 - 1 2 0}$ days after birth & Use in infants $\mathbf{0 - 1 2 0}$ days after birth \\
Any macrolide & $30091(100)$ & $21557(100)$ & $6591(100)$ \\
\hline Erythromycin & $25240(83.9)$ & $11146(51.7)$ & $5372(81.5)$ \\
\hline Roxithromycin & $4193(13.9)$ & $6614(30.7)$ & $2(0.03)$ \\
\hline Azithromycin & $6757(22.5)$ & $3947(18.3)$ & $425(6.4)$ \\
\hline Clarithromycin & $930(3.1)$ & $1030(4.8)$ & $855(13.0)$ \\
\hline Spiramycin & $193(0.6)$ & $1(<0.01)$ & - \\
\hline$>1$ macrolide subtype & $1386(4.6)$ & $1151(5.3)$ & $63(0.96)$
\end{tabular}

*Since a cohort member could have used more than one macrolide subtype, percentages for individual subtypes add up to more than $100 \%$. 
Table 2| Maternal and infant use of macrolides by characteristics of a cohort of singletons born 1996 to 2011, Denmark. Values are percentages (numbers) of person years of follow-up unless stated otherwise

Maternal and infant use of macrolides

\begin{tabular}{|c|c|c|c|c|c|}
\hline \multirow[b]{2}{*}{ Characteristics } & & \multirow[b]{2}{*}{$\begin{array}{l}\text { All cohort members } \\
\text { ( } n=999378,326805 \\
\text { person years) }\end{array}$} & \multirow[b]{2}{*}{$\begin{array}{c}\%\left(\mathrm{No}^{\star}\right) \text { of IHPS } \\
\text { cases }(n=880)\end{array}$} \\
\hline & $\begin{array}{l}\text { Maternal use during } \\
\text { pregnancy ( } n=30091 \text {, } \\
9855 \text { person years) }\end{array}$ & $\begin{array}{l}\text { Maternal use } 0-120 \text { days } \\
\text { after birth ( } n=21557,3917 \\
\text { person years) }\end{array}$ & $\begin{array}{l}\text { Use in infants } 0-120 \text { days } \\
\text { after birth }(n=6591,991 \\
\text { person years }\end{array}$ & & \\
\hline \multicolumn{6}{|l|}{ Calendar years: } \\
\hline 1996-99 & $30.9(3041)$ & $27.2(1066)$ & $25.2(249)$ & $25.1(82$ 122) & $23.6(208)$ \\
\hline 2000-03 & $26.9(2654)$ & $25.6(1002)$ & $29.3(291)$ & 25.4 (82 988) & $25.2(222)$ \\
\hline 2004-07 & $23.5(2311)$ & 24.7 (967) & $25.1(248)$ & $25.0(81839)$ & $27.1(238)$ \\
\hline $2008-11$ & $18.8(1849)$ & $22.5(882)$ & $20.4(202)$ & $24.4(79856)$ & $24.1(212)$ \\
\hline
\end{tabular}

Current age of infant

(weeks):

\begin{tabular}{llcccc}
\hline $0-2$ & $17.5(1724)$ & $3.7(143)$ & $1.9(19)$ & $17.5(57239)$ & $15.9(140)$ \\
\hline $3-12$ & $57.9(2427)$ & $56.2(2200)$ & $52.8(523)$ & $57.9(189184)$ & $83.4(734)$ \\
\hline $13-17$ & $24.6(5704)$ & $40.2(1573)$ & $45.3(449)$ & $24.6(80382)$ & $0.7(6)$ \\
\hline
\end{tabular}

Maternal age at birth

(years):

\begin{tabular}{|c|c|c|c|c|c|}
\hline$\leq 24$ & $17.8(1753)$ & $16.1(632)$ & $20.3(201)$ & $13.8(44973)$ & $17.3(152)$ \\
\hline $25-34$ & $64.3(6333)$ & $67.1(2630)$ & $66.4(657)$ & $69.5(227216)$ & $67.1(590)$ \\
\hline $35-44$ & $17.9(1759)$ & $16.7(653)$ & $13.3(131)$ & $16.6(54357)$ & $15.6(137)$ \\
\hline$\geq 45$ & $0.1(10)$ & $0.1(3)$ & $<0.1(<1)$ & $0.1(259)$ & $0.1(1)$ \\
\hline First born & $36.3(3576)$ & $40.0(1565)$ & $35.3(350)$ & $43.4(141946)$ & $56.1(494)$ \\
\hline Boy & $51.2(5048)$ & $51.8(2028)$ & $54.4(539)$ & $51.3(167561)$ & $85.6(753)$ \\
\hline Preterm birth $\dagger$ & $5.0(201)$ & $4.3(74)$ & $3.5(17)$ & $5.6(18384)$ & $7.6(67)$ \\
\hline Small for gestational age $\ddagger$ & $11.2(1103)$ & $10.5(410)$ & $9.8(97)$ & $10.5(34446)$ & $14.0(123)$ \\
\hline $\begin{array}{l}\text { Delivered by caesarean } \\
\text { section }\end{array}$ & $18.5(1818)$ & $21.1(826)$ & $14.7(145)$ & $17.3(56414)$ & $25.0(220)$ \\
\hline Maternal smoking§ & $26.5(2609)$ & $24.0(938)$ & $21.5(213)$ & $18.1(59$ 183) & $26.9(237)$ \\
\hline $\begin{array}{l}\text { Major congenital } \\
\text { malformations }\end{array}$ & $2.3(227)$ & $2.3(91)$ & $2.8(28)$ & $2.2(7192)$ & $5.6(49)$ \\
\hline
\end{tabular}

IHPS=infantile hypertrophic pyloric stenosis.

For all three categories of maternal and infant use of macrolides, those with missing values for covariates did not contribute person years of follow-up, whereas person years of follow-up for all cohort members reflects all infants born in Denmark during study period. Among all cohort members covariates with missing values were: gestational age $(0.9 \%)$, small for gestational age $(0.6 \%)$, caesarean section $(0.6 \%)$, and maternal smoking ( $3.9 \%)$. One person year of follow-up covers one cohort member followed for one year, and percentage of person years of follow-up can be interpreted as the distribution of individuals in the cohort.

${ }^{*}$ Number of cohort members.

†Delivery before gestational age 37 weeks.

‡Lowest 10th centile of gestational age specific birth weight.

§Includes mothers who reported active smoking during entire or part of pregnancy.

IAmong the 49 cases of IHPS with a congenital malformation (before or on same day as diagnosis of IHPS), 15 were gastrointestinal malformations. No cases of abdominal wall defects occurred among the 49 cases. 
Table 3 | Rate ratios of infantile hypertrophic pyloric stenosis (IHPS) by maternal and infant use of penicillin $\mathrm{V}$ or amoxicillin during pregnancy and up to 120 days after birth in a cohort of 999378 singletons, 1996 to 2011

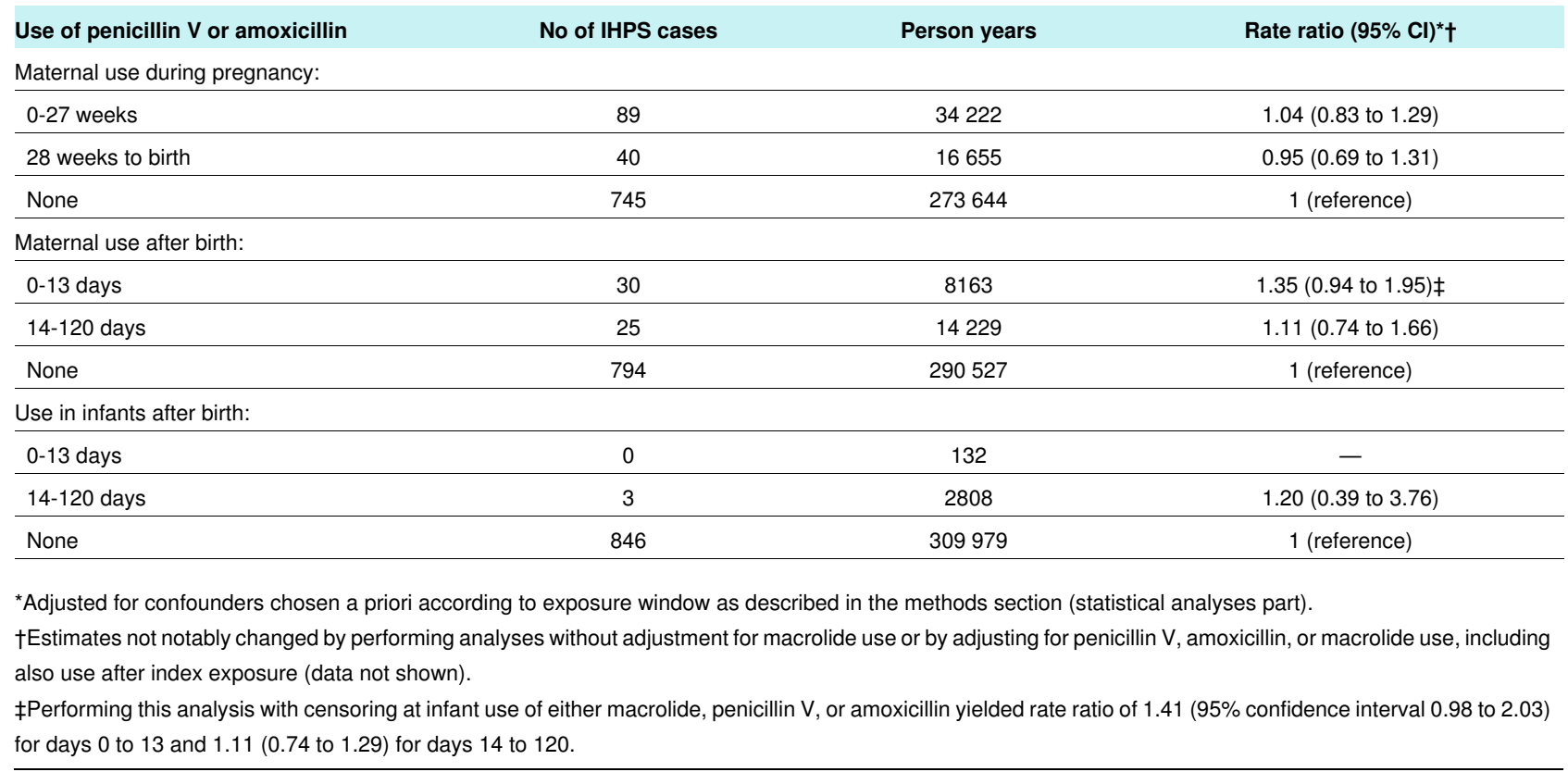




\section{Figure}

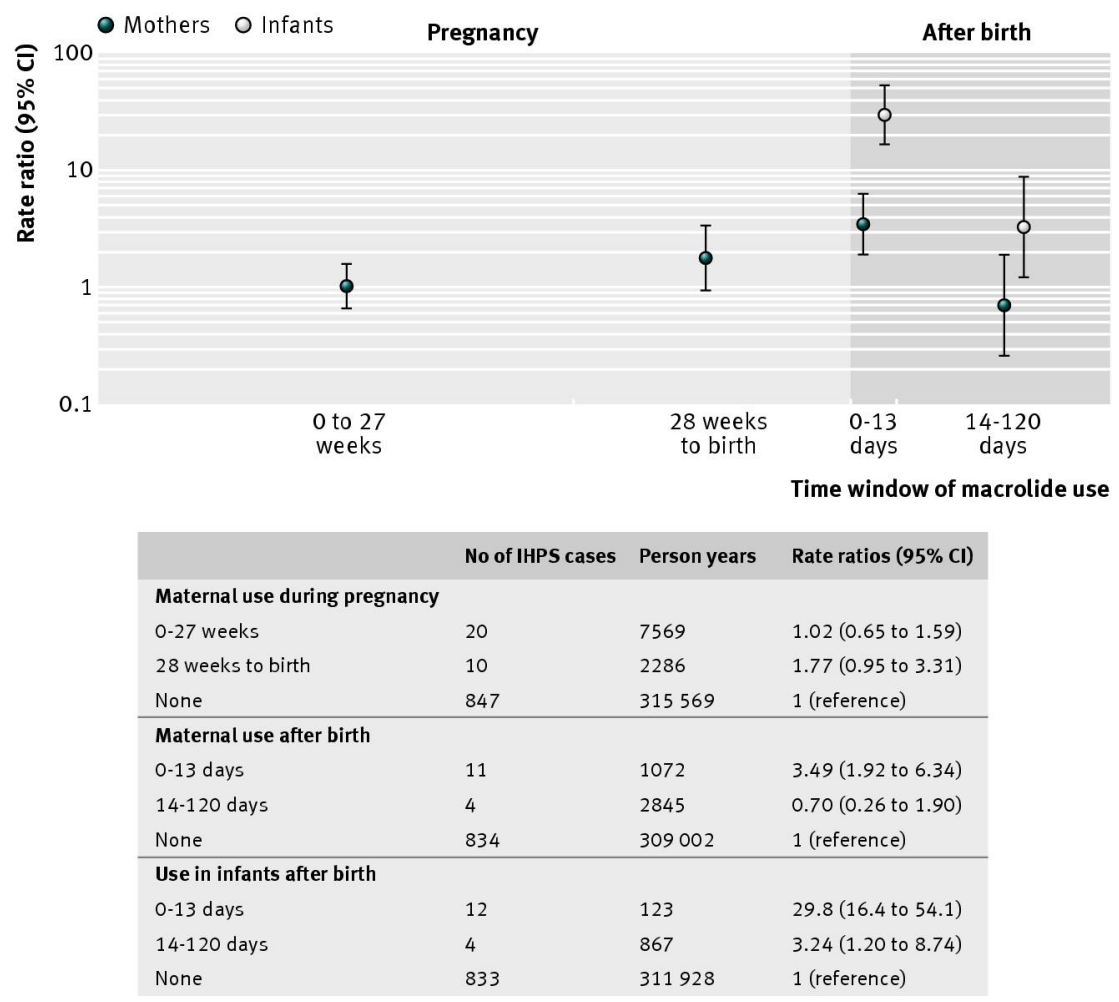

Rate ratios of infantile hypertrophic pyloric stenosis according to maternal and infant use of macrolides during pregnancy and up to 120 days after birth in a cohort of 999378 singletons. Log scale used for rate ratio. Rate ratios were adjusted for confounders chosen a priori according to exposure window as described in the methods section (statistical analyses part). $P$ for homogeneity: maternal use during gestational weeks 0-27 v gestational weeks 28 to birth, $P=0.15$; maternal use day $0-13 v 14-120, P=0.007$; use in infants day $0-13 v 14-120, P<0.001$. Results were similar without adjustment for previous use of macrolides (see supplementary table 2 ) 